\title{
Magnetically Enhanced Inductive Source for Electric Propulsion Applications
}

\author{
J.E. Foster ${ }^{*}$ and Eric Gillman, ${ }^{\dagger}$ Brad Summers ${ }^{\ddagger}$ and Brandon Weatherford ${ }^{\S}$ \\ The University of Michigan, Ann Arbor, 48105
}

\begin{abstract}
[Abstract] A magnetically enhanced inductive discharge was investigated for electric propulsion applications. The high plasma density produced by the source makes it attractive as an ion source for a gridded ion thruster or possibly a stand-alone ambipolar thruster. Plasma is produced by a compact "stovetop", spiral antenna similar to that used in plasma processing sources. Operation on Argon to pressures as low as 1 mTorr were demonstrated at powers ranging from 100 to $250 \mathrm{~W}$. Ion current as high as $1 \mathrm{~A}$ were extracted from the 10 cm diameter device. Plasma properties and ion production efficiency are reported and commented upon.
\end{abstract}

\section{Nomenclature}

$A_{\text {eff }} \quad=$ effective loss area

$B \quad=$ magnetic field

$E \quad=$ electric field

$e \quad=$ elementary charge of an electron

$\varepsilon_{T} \quad=$ energy loss per ion lost to the wall

$\mu_{o} \quad=$ permeability of free space

$\mu \quad=$ permeability in medium

$\mathrm{d} t=$ time step

$m \quad=$ mass of an electron

$n_{s} \quad=$ plasma density

$P_{a b s} \quad=$ absorbed power

$T_{e} \quad=$ electron temperature

$u_{B} \quad=$ Bohm velocity

$\delta \quad=$ skin depth

$\sigma \quad=$ electrical conductivity

$\omega=\mathrm{rf}$ angular frequency

\section{Introduction}

$\mathrm{R}$ ADIO frequency driven plasma sources have seen success in electric propulsion applications as embodied in the RIT series of ion thrusters. ${ }^{1}$ Recently, a radio frequency ion thruster originally intended for north-south station keeping was used for orbit-raising to salvage a communication satellite (ARTEMIS) accidentally placed in the wrong orbit. ${ }^{2}$ Rf-driven plasma production offers many distinct advantages over conventional, DC plasma sources presently being used in electric propulsion devices such as ion and Hall thrusters. These DC engines feature the

\footnotetext{
*Associate Professor, Dept. Nuclear Engineering, University of Michigan, AIAA Member.

${ }^{\dagger}$ Graduate Student, Dept. Nuclear Engineering, University of Michigan.

* Graduate Student, Dept. Nuclear Engineering, University of Michigan.

${ }^{\S}$ Graduate Student, Dept. Nuclear Engineering, University of Michigan.
} 
hollow cathode as the electron source for ion production and beam neutralization. Hollow cathodes are subject to physical and thermochemical wear that ultimately limits their lifetime to roughly $30 \mathrm{kHrs} .{ }^{3}$ On the other hand, electrodeless systems such as rf and microwave plasma thrusters do not suffer from such wear out mechanisms. The ionizer stage in these systems is limited primarily by the lifetime of the power supply itself. Microwave power supplies have demonstrated on orbit life of over $140 \mathrm{kHr} .{ }^{4}$ Flight demonstration of the capability and potential of microwave ion thrusters has been validated on the Hayabusa asteroid rendezvous mission. ${ }^{5}$ These microwave thrusters join the NSTAR (Deep Space 1) ${ }^{6}$ and PPS1350 (SMART 1) ${ }^{7}$ as electric propulsion systems utilized on deep space missions. The Hayabusa thrusters to date have accumulated over 25000 hours on in-space operation time (distributed among four thrusters). Unlike microwave thrusters, rf systems do typically utilize a conventional hollow cathode for beam neutralization. The neutralizer, however, is not immersed in a hostile plasma environment such as that of a discharge cathode which undergoes constant ion bombardment; therefore, the neutralizer is expected to undergo only limited wear provided it does not intersect the beam.

In contrast to traveling wave tube efficiencies, lower frequency rf power supplies have higher electrical efficiencies, typically comparable to DC sources. Therefore, rf driven thrusters from an efficiency standpoint can fill the need for long life thruster systems in the low to medium power range (few hundred Watts to several $\mathrm{kW}$ ). Conventional rf thruster systems employ the classic inductive discharge configuration. ${ }^{8}$ Here an external, rf driven coil wrapped along the outer diameter of a dielectric discharge chamber is used to couple power to the gas to produce and sustain a plasma discharge by transformer action. In this regard, in these "barrel" sources, power is deposited into a skin depth typically on the order of centimeters in spatial extent (collisionless case). Density and power dissipation is highest in this skin depth layer. This follows directly from the solution to the wave equation where the skin depth represents the spatial decay constant of a damped wave in a conductive medium. ${ }^{9}$ In recent years, the move toward high-density plasma sources for plasma processing application has made significant advances in the improvement of inductive plasma sources. A review of such advances may be found in Hopwood. ${ }^{10}$ These advances include movement away from the "barrel"-type inductive sources to the planar, "stove top" coil design featuring multipole confinement and Faraday shielding to mitigate sputtering effects due to capacitive coupling. The planar coil designs allow for very large area, low aspect ratio configurations. Such a source can be used as an ionizer chamber for an ion thruster allowing for very compact thruster designs. The planar coil design allows for not only scaling to larger thruster diameters, but the relative low aspect ratios may mitigate some issues associated with vibration qualification as well. The planar spiral inductive discharge is capable of producing very high plasma densities (well into $\left.10^{12} \# / \mathrm{cm}^{3}\right)$ with low plasma potentials $(<20 \mathrm{~V})$ at reduced pressures. Unlike the barrel inductive sources, the planar configurations are amenable to multipole confinement schemes utilizing permanent magnets. The use of such arrangement improves not only density at reduced pressure but also uniformity and discharge efficiency. ${ }^{11}$ Finally, because the plasma heating and production occurs within a few skin depths downstream of the antenna, the issue of eddy current induction/heating of the grids can be completely avoided by simply utilizing discharge chamber depths larger than the skin depth. Additionally, the compactness of the coil mitigates the need for extensive shielding. On the surface, it would appear that the planar, spiral source is the natural evolutionary step for inductive ion sources for electric propulsion. Based on the promising attributes of the flat spiral antenna sources as well as the demonstrated performance of the sources in plasma processing systems up to $300 \mathrm{~mm}$ in diameter with high uniformity, an investigation was initiated to assess the feasibility of using a flat spiral type inductive source for electric propulsion applications. These attributes that have led us to investigate the planar spiral antenna ionizer as an inductive source specifically for ion thruster applications as well as a possibly stand alone plasma source for ambipolar diffusion class ion thruster systems ${ }^{12,13}$.

In this work, the variation in plasma density, plasma temperature and extractable ion current as a function of power and pressure in a magnetically enhanced inductive source is discussed. Estimates of ion production efficiency will be calculated and compared with compatible data acquired in the open literature. The applicability of an inductive ion source featuring the planar spiral antenna geometry for electric propulsion applications will be commented upon.

\section{Background and Experimental Procedure}

\section{A. Basic Physics}

Inductive discharge breakdown and discharge maintenance is due to transformer action between the antenna and the secondary coil, the plasma, as described by Faraday's law: 


$$
\nabla \times \vec{E}=-\frac{d \vec{B}}{d t}
$$

Here, an electric field is produced in the discharge chamber by the action of the time varying magnetic field produced by rf current circulating in the planar coil. At low pressures, the penetration depth of the electric field depends on the density:

$$
\delta=\sqrt{\frac{m}{e^{2} \mu_{o} n_{s}}}
$$

Here, the low-pressure limit is defined at conditions where the excitation frequency greatly exceeds the electron neutral collision frequency. The operating rf frequency used in this work is $13.56 \mathrm{MHz}$. A neutral collision frequency equivalent to this excitation frequency occurs around $25 \mathrm{mTorr}$. Measurements were acquired at pressures considerably below this value. For reference, assuming collisionless conditions prevail, at a plasma density of $10^{11} \# / \mathrm{cm}^{3}$, the skin depth is nearly $2 \mathrm{~cm}$. For the discharge to operate efficiently, the discharge chamber depth therefore must be at least a depth or so deep; therefore very shallow discharge chambers are possible. Figure 1 schematically depicts the compact planar coil along with the induced electric field produced by the time varying magnetic field which is in turn is produced by the rf driven currents in the coil. In this manner power is transferred across the quartz vacuum window into the plasma.

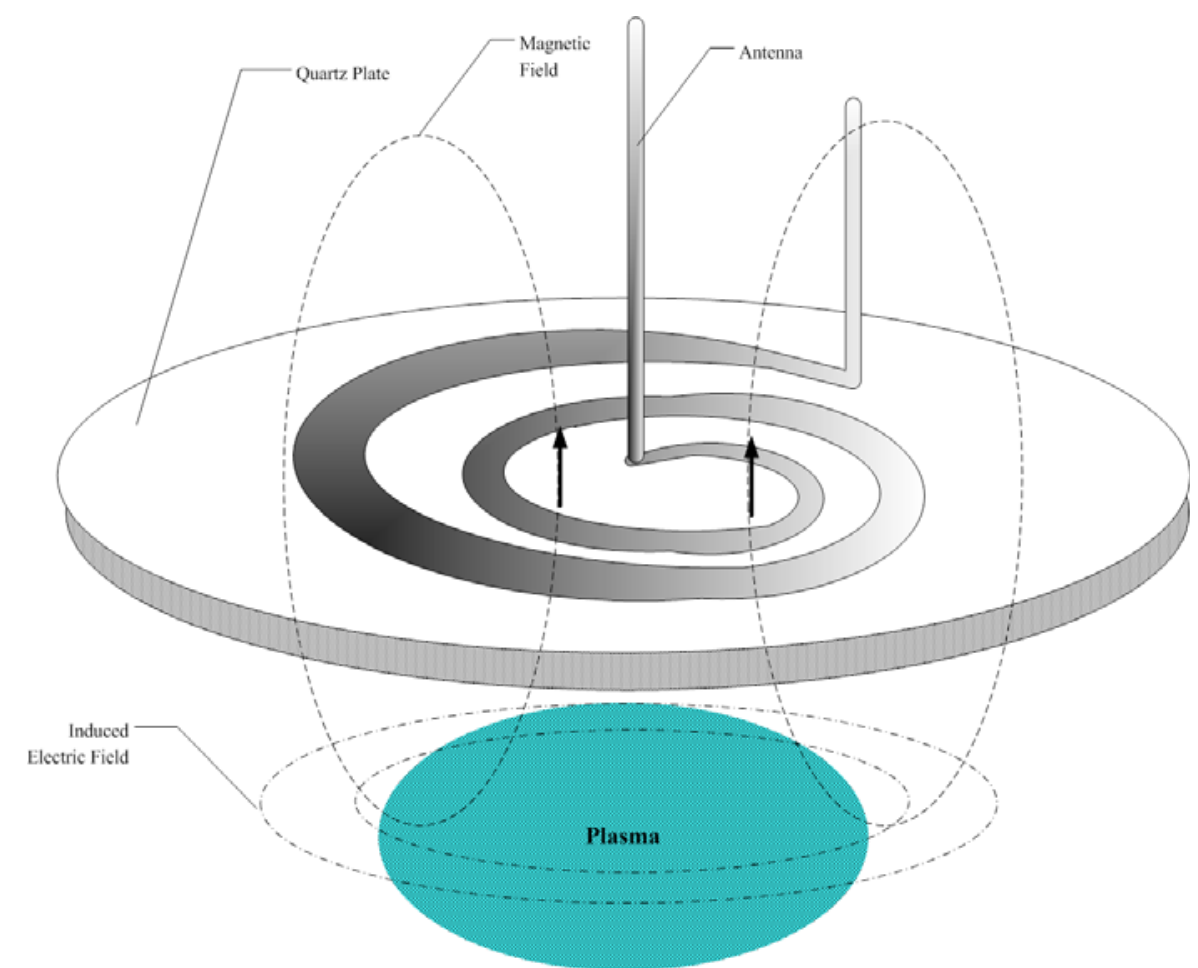

Figure 1. Schematic depiction of planar, compact antenna and induced electric field.

It is well known that multipole configurations can extend the operating range of a plasma source to include lower pressure while still maintaining high uniformity. ${ }^{14}$ In the work presented here, a ring cusp magnetic section is integrated with a planar inductive source to improve plasma density at reduced pressures. The magnetic circuit reduces diffusion losses to the wall and extends the residence time of the electrons in the heating zone. These contained hot electrons improve the overall ionization efficiency. It should be pointed out that even for plasma processing applications, very high electrical efficiencies for planar inductive sources have been reported. For example, Keller reports $80-90$ percent electrical efficiencies. ${ }^{15}$ Here, efficiency is defined as the ratio of plasma power (plasma production) to total input electrical power.

For ion thruster applications, it is expected that some backsputtered material from low-level grid erosion or backsputtered material from test facility sputtering could deposit onto the rf quartz coupling plate. Such deposition 
would be minimal in space provided the grids are properly aligned and gapped. The effect of the coating would be that of attenuation of the power actually deposited into the plasma. The problem of coatings on the dielectric window for inductive sources can be addressed through the use of a Faraday shield which can act as a baffle or shadow shield to prevent coatings from occurring on the window. The Faraday shield therefore plays this role in addition to the role of shorting out capacitive fields. Hopwood and colleagues have demonstrated the utility of the Faraday shield to defeat the coating problem in metal vapor plasmas. ${ }^{16}$ Here, deposition vapor rates of $\sim 40 \mathrm{~nm} / \mathrm{min}$ were investigated in the presence of a Faraday shield. These metal vapor deposition rates were considerably higher than that which would be expected from space or ground testing. The amount of attenuation expected for a system without the Faraday shield depends on the ratio of the conductive film thickness to the skin depth. For the case of an electromagnetic plane wave incident on a metal, the penetration depth is on the order the wavelength in the conductor:

$$
\delta=\sqrt{\frac{2}{\mu \sigma \omega}}
$$

At $13.56 \mathrm{MHz}$, the wavelength inside carbon, a typical grid material, with an electrical conductivity of $61,000 \mathrm{ohm}-$ $\mathrm{m}$, is of order $1 \mathrm{~mm}$, suggesting that attenuation effects should be minimal as the ratio of skin depth to 1 micron, a typically observed thickness observed after long duration ion thruster wear testing, ${ }^{17}$ is of order 1000 . Lower frequency operation, $1 \mathrm{MHz}$ for example, would result in even less attenuation. RIT thrusters, which operate around $1 \mathrm{MHz}$ and have been wear tested as well as flight tested, demonstrate that deposition of metal coatings typically observed on ion thruster walls on the dielectric discharge chamber is not an issue. ${ }^{18}$

\section{B. Experimental Set-up}

The experimental investigation took place in a Gaseous Electronics Reference Cell. The test cell was specifically designed as a reference apparatus for reproducible experiments in plasma processing research. ${ }^{19}$ The exact dimensions of the apparatus may be found in reference 15 . The discharge chamber itself is roughly $30 \mathrm{~cm}$ in diameter and about $30 \mathrm{~cm}$ in height. The apparatus is evacuated with a turbomolecular pump with a pumping speed of $400 \mathrm{l} / \mathrm{s}$. Ultimate base pressures around $10^{-7}$ Torr are achievable with this configuration. During normal testing, gas is input using a precision mass flow controller. The gas is actually input into the bell jar, which in turn flows into the source region by diffusion. In this case, the pressure inside the source should reflect the pressure in the bell jar as measured using ionization and thermocouple gauges attached to the facility. At a given flow rate, test cell pressure is adjusted using a variable aperture throttle valve located upstream of the turbomolecular pump. For the experimental results presented here, argon gas was used. For the data presented here, the throttle valve upstream of the turbo-pump was set at 12 percent open. Figure 2 depicts the GEC bell jar.

The rf antenna consisted of a $0.32 \mathrm{~cm}$ diameter copper planar-spiral coil antenna adjacent to a $2.5 \mathrm{~cm}$ thick quartz window. Rf power is coupled into the discharge chamber across this window. The antenna itself is coupled to a $500 \mathrm{~W} 13.56 \mathrm{MHz}$ rf power supply through a Pi configured matching network. ${ }^{22}$ The matching network can be set to automatically match the impedance of the power supply to the load. Typically during a test, the impedance is matched and then the auto-tuner is shut off. Once matched, the load absorbs most of the input power over a wide power and pressure range. In all cases, reflected power was less than 5\% of input power. The inset in Figure 2 depicts the inductive plasma source nominally operating without magnetic confinement. 


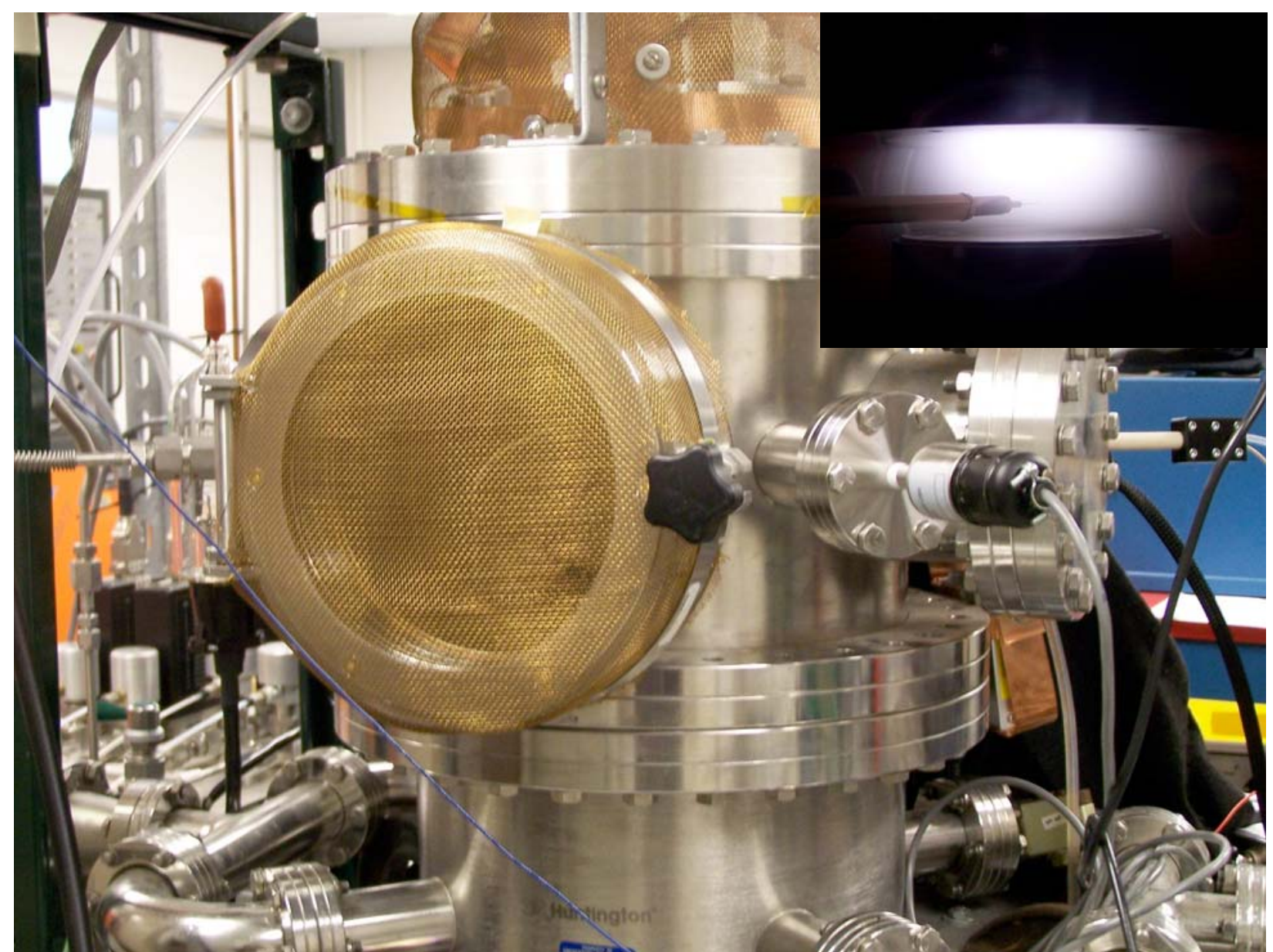

Figure 2. GEC test cell. Note inset depicting discharge operation without magnetic enhancement. Rf compensated Langmuir probe also depicted.

As shown in Figure 3, located just downstream $(\sim 1 \mathrm{~cm})$ of the quartz coupling window was a mild steel, split ring cylinder containing a series of magnets of alternating polarity. This ring-cusp magnetic circuit middle section was used to confine the plasma and improve the ionization efficiency of the discharge. To improve the uniformity of the ring cusp circuit, metal straps, held magnetically in place, were used to jumper portions of the split ring. The jumpers make good magnetic contact but poor electrical contact at $13.56 \mathrm{MHz}$. This minimizes eddy losses in the magnetic container. Located just downstream of the cylindrical section (essentially terminating the exit plane, $\sim 40$ $\mathrm{mm}$ downstream of the quartz window) was a $10 \mathrm{~cm}$ diameter bias-able electrode. This electrode was typically biased around $-38 \mathrm{~V}$ to collect ion saturation current to assess ion flux exiting the discharge chamber. Inserted onto centerline of the apparatus was a cylindrical Langmuir probe. The probe tip was $1 \mathrm{~cm}$ long with a total surface area of 1 square centimeter. It was used to acquire plasma density, electron temperature, plasma potential, and floating potential in the middle of the discharge. Because the plasma potential oscillates harmonically at the rf frequency, the potential difference between the probe tip and the plasma potential changes with time. This gives rise to a distorted Langmuir probe IV characteristic. To offset this an inline filter and reference probe is used to correct the IV characteristic. By increasing the impedance of the probe tip to ground at the rf frequency and harmonics, the probe tip will follow the oscillations in the plasma. As long as these oscillations are less than the $T_{e} / e$ in magnitude, the analysis of the traces follows that of a probe in a DC plasma. ${ }^{20}$ The inline filter and associated circuitry achieves this end. The probe was located approximately $20 \mathrm{~mm}$ below the quartz window. In all cases the probe was at least one skin depth away from quartz plate. A photograph of the probe without the discharge magnetic insert installed is shown in Figure 2. Corrections to the probe's IV characteristic to account for magnetic effects are neglected as the Larmor radius at the tip location was much larger than the probe radius. 
The discharge is typically initiated by increasing the pressure to around 10 mTorr and then increasing power until the oscillating electric field is sufficiently high that production matches diffusion losses. This typically occurred at very low powers $(\sim<10 \mathrm{~W})$. Here a self-sustaining plasma is formed. Once formed it is possible to maintain the inductive discharge to pressure slightly below 1 mTorr. At each change in flow, the pressure is allowed to stabilize before data is acquired. In the cases presented here, the amount of reflected power was less than 5 percent and did not vary appreciably as a function of flow and pressure.

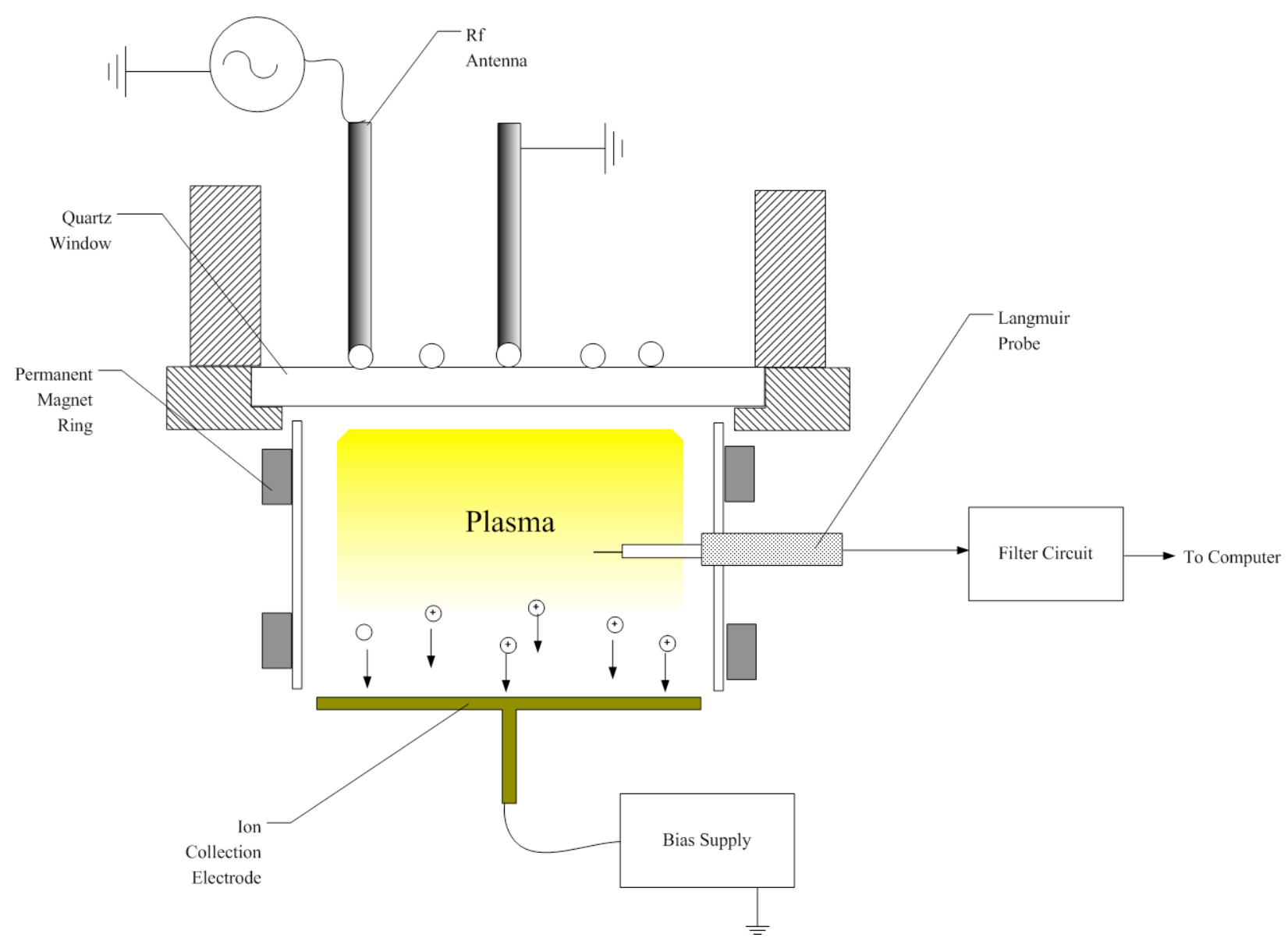

Figure 3. Schematic depiction of the experimental set-up.

\section{Results}

This investigation aimed to look at the ion production capability of a planar inductive source retrofitted with a magnetic multipole jacket to enhance ionization efficiency particularly at low pressures. The source achieved this end allowing for a $10 \mathrm{X}$ reduction in operating pressure allowing for operation in the target range of 1-2 mTorr possible. The jacket also served to confine the plasma making the configuration more thruster-like. In contrast, typical plasma processing reactors do not utilize plasma limiters as it would be a contamination source. The performance of the source was assessed by characterizing the plasma properties such as plasma density and electron temperature at the center of the source. An upper limit on the maximum extractable ion current was assessed by collecting ion saturation current at a biased electrode located at the exit plane of the source. With this information, expected ion production costs could be estimated. The data presented is with argon as the working gas. It is expected that performance should improve considerably with subsequent testing utilizing xenon, owing to the lower ionization potential. For example, in the development of the RIT 10 thrusters, it was found that to attain discharge 
performance (W/A) similar to xenon, it was necessary to operate the discharge chamber on argon at much higher pressures $(\sim 2 \mathrm{X}$ in cases $) .^{21}$

\section{A. Plasma Density}

Figure four depicts the variation in plasma density as a function of pressure all the way down to 1 mTorr. As can be seen in the Figure, at fixed power, plasma density increases monotonically as a function of pressure. The rate of density increase with pressure depends on power. The lowest power case investigated, $100 \mathrm{~W}$, indicates a considerably smaller slope than the higher power cases. Also worth noting, as expected, plasma density at fixed pressure increased with power. This increase was most notable at pressures above 2 mTorr. Below 2 mTorr, the difference in plasma density at $100 \mathrm{~W}$ and $170 \mathrm{~W}$ was not appreciable, suggesting that at lower powers, at a fixed pressure, the ionization efficiency saturates. In general, if one considers the rf breakdown characteristic for inert gases, the required breakdown field tends to increase sharply with decreasing pressure particularly at collision frequencies well below the rf frequency. This is due to the infrequency of ionization collisions. As the electric field increases, the energy that electron can gain and impact a neutral at a given instant is higher. The observed behavior in the $100 \mathrm{~W}$ to $170 \mathrm{~W}$ range is likely due to a combination of reduced electric field and consequently electron energy projected onto the ionization and excitation cross sections for argon. Unlike the $250 \mathrm{~W}$ case, similarities in density at the low pressures at the $100 \mathrm{~W}$ and $170 \mathrm{~W}$ cases indicate that plasma production at these two powers may be dominated by capacitive rather than inductive field driven ionization. The data does imply that at least low pressures, operating at $100 \mathrm{~W}$ rather than $170 \mathrm{~W}$ may be more efficient, that is, if the ion extraction takes place at the plane of the Langmuir probe.

The plasma densities obtained in this work can be compared with conventional GEC planar inductive source without magnetic enhancement. Miller et al characterized inductive plasma variations of a GEC cell down to 5 mTorr. $^{22}$ Plotted on Figure 4 are plasma density measurements acquired at similar locations acquired in this past work. As can be seen in the figure, the magnetic enhancement, at a given power, boosts the density considerably thereby allowing for operation at reduced power and lower pressure.

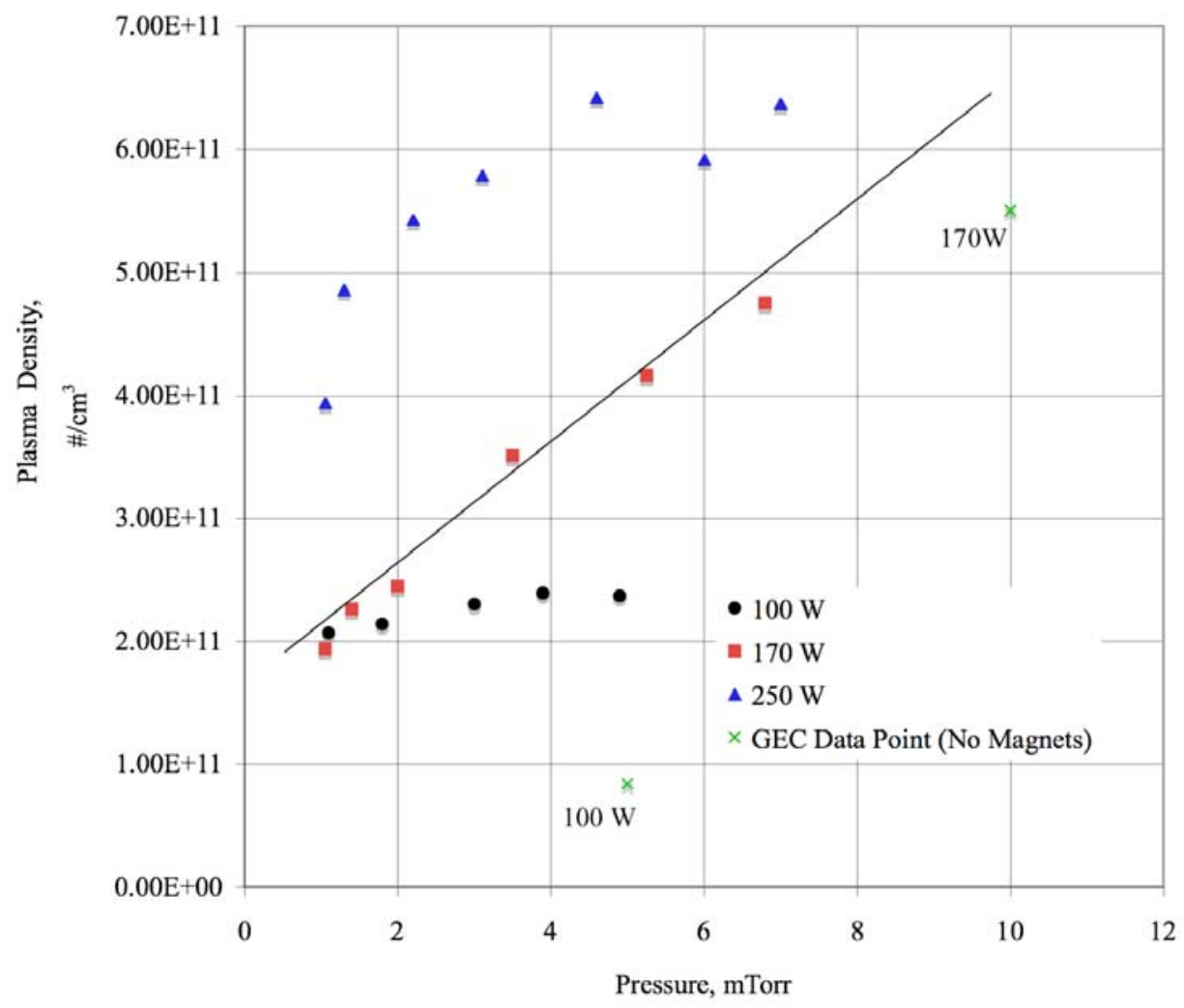

Figure 4. Plasma density variations as a function of chamber pressure at different input powers. 


\section{B. Electron Temperature}

Figure 5 illustrates variations in the measured electron temperature as a function of pressure. Typically for electropositive discharges such as this one, the electron temperature is a strong function of the background pressure. It is expected that the electron temperature decrease with increasing pressure. Strictly speaking this behavior was observed the clearest at $170 \mathrm{~W}$. The electron temperature at the low power condition was fairly constant at a high value to $7.6 \mathrm{eV}$. This may be indicative of increased capacitive coupling at this low power condition. The electron temperature at the $250 \mathrm{~W}$ case at the lower pressures was around $6 \mathrm{eV}$ and actually increases somewhat above 5 mTorr. For reference, two higher pressure points from a conventional GEC cell are shown. Here it can be seen that these points did not vary appreciably with power as is typical for conventional electropositive discharges. ${ }^{9}$ In the case of the magnetically enhanced system, the spread in temperature with power is significant. Additionally, the GEC points do not appear to lie on any of the family of curves acquired from the magnetically-enhanced source. The difference in ordering and magnitude of the average electron temperature at a given pressure and varying powers at present is not well understood though it is speculated the capacitive coupling plays a role perhaps in a complicated way as the electrons are also interacting with the magnetic field in the presence of the capacitive electric field.

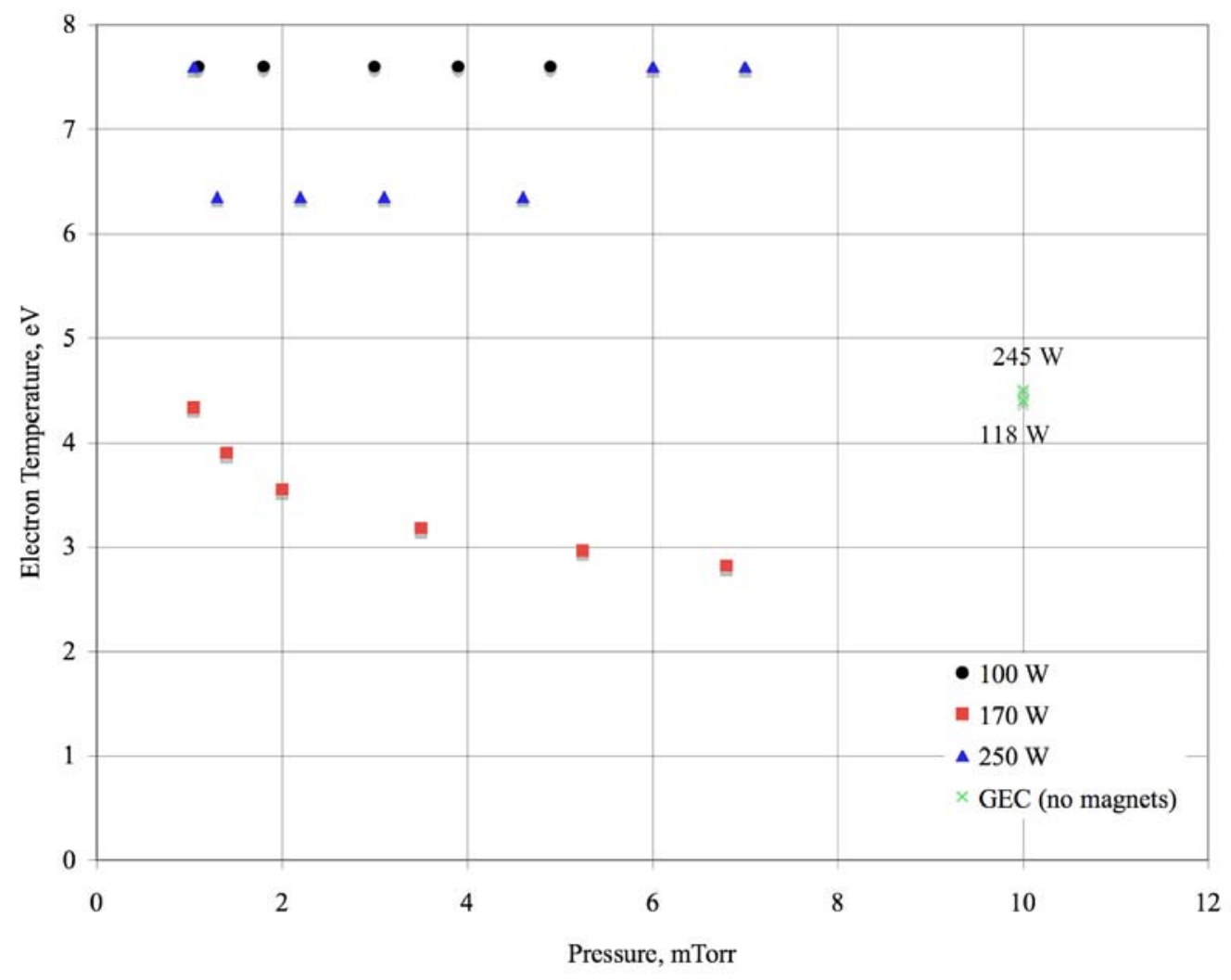

Figure 5. Variation in the electron temperature with pressure and power.

\section{Collected ion current}

Of great merit to the magnetically enhanced source is the extractable ion current and the ion production efficiency. The extractable ion current was estimated by biasing a $10 \mathrm{~cm}$ disk electrode located $44 \mathrm{~mm}$ downstream of the window to ion saturation $(-38 \mathrm{~V})$. This data was also acquired as a function of pressure and power. Figure 6 depicts these variations. Typical ion current behavior is illustrated in the Figure. At a fixed pressure, the current increased with input power following plasma density. Indeed the data appears to suggest that the current is directly proportional to the power. Figure 7 illustrates the ratio of the ion current taken at 250 
$\mathrm{W}$ to that acquired at lower powers. In typical electropositive discharges, the ion flux scales with the plasma density and the square root of the electron temperature. Mathematically, the density scales as: ${ }^{9}$

$$
n_{s}=\frac{P_{a b s}}{e u_{B} A_{e f f} \varepsilon_{T}}
$$

The loss area is determined by the magnetic circuit and the electron temperature. One would then expect to first order the ratio of $250 \mathrm{~W}$ ion current to $100 \mathrm{~W}$ ion current to be approximately 2.5 , following the power ratio. But instead it was measured to be $40 \%$ higher at approximately a factor of 3 for higher pressures and increased at the lower pressures indicating that the lower power discharge were not as efficient. At $170 \mathrm{~W}$ on the other hand, the ratio is expected to be 1.5 and indeed over the broad range it is in fact approximately 1.5 . Deviations do occur however at the lower pressures suggesting that power coupling efficiency may be the issue. In general, all inductively coupled discharges possess some inherent capacitive coupling. At lower powers in particular, the capacitive coupling becomes more pronounced due to the fact that the plasma production via high voltage sheath acceleration begins to dominate rather than production by inductive electric fields. Its only when the inductive electric field is sufficiently high that this mode becomes the dominant source of plasma production, though the lower power contributor, the capacitive variant, never actually goes away. So in this case at the lower powers and lower pressures, it becomes increasingly difficult to couple power into the plasma inductively. Indeed, the magnetic insert section was implemented to improve such coupling. Because the capacitive contribution is not as efficient as one whose inductive power fraction is considerably higher, the current ratio can be expected to be higher at the lower powers and pressures. Again, this is due to the energy loss per ion lost to the wall term. This is higher for capacitive discharges because relatively speaking the power lost to the walls in a capacitive discharge (sheath voltage $\sim 100 \mathrm{~s} \mathrm{~V}$ ) is large compared to inductive discharges $(\sim 10 \mathrm{~V})$. This explains not only the deviations in the ratio at the lower powers, but also the deviations appearing at the lower pressures. It may be possible to improve efficiency at the lower powers and pressures by improving the magnetic circuit, which at present is not optimized.

Also worth pointing out in Figure 6 is that the ion current does appear to saturate at the higher pressures, remaining relatively constant above some threshold pressure. Below this threshold pressure, there is a precipitous drop in current with decreasing pressure. The location of the knee demarcating the current drop-off shifts with operating power, changing from $2.75 \mathrm{mTorr}$ at $100 \mathrm{~W}$ to 1.5 mTorr at $250 \mathrm{~W}$. Again, a goal of this work is to displace the knees to lower pressure using magnetic containment. It is expected that the knees will also shift to lower pressure if the source were to be operated on xenon gas. Also as can be seen in the figure, it was possible to extract nearly $1 \mathrm{~A}$ of current at $250 \mathrm{~W}$ and 3 mTorr with argon!

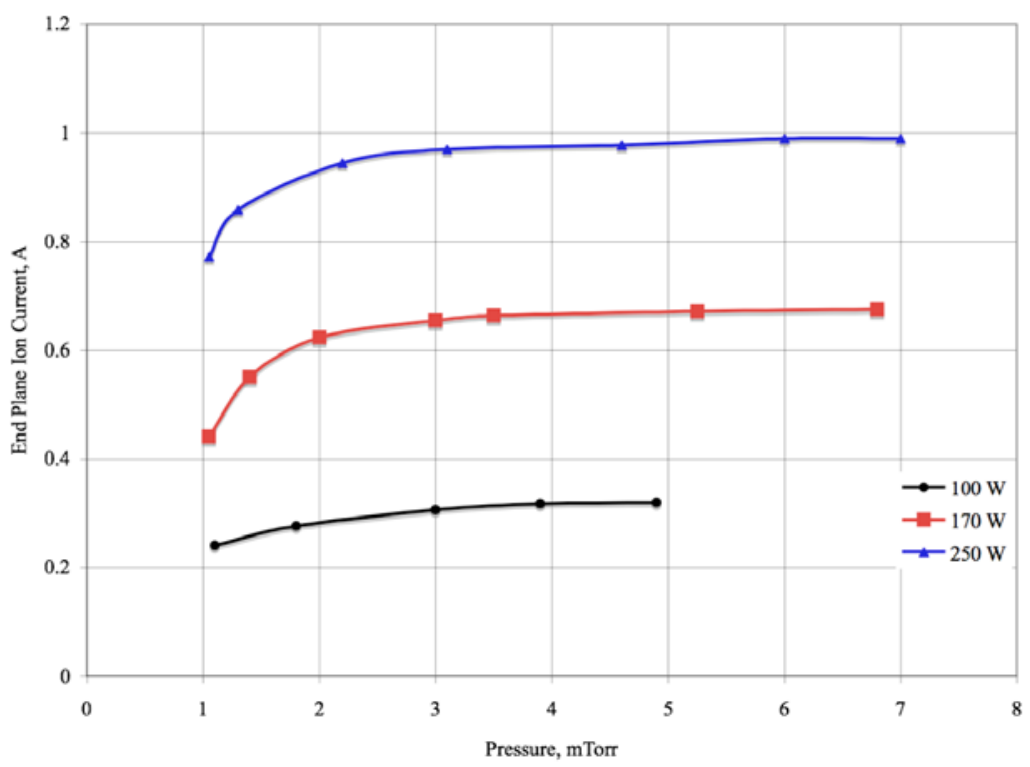

Figure 6. End plane ion saturation current collected as a function of pressure and power. 


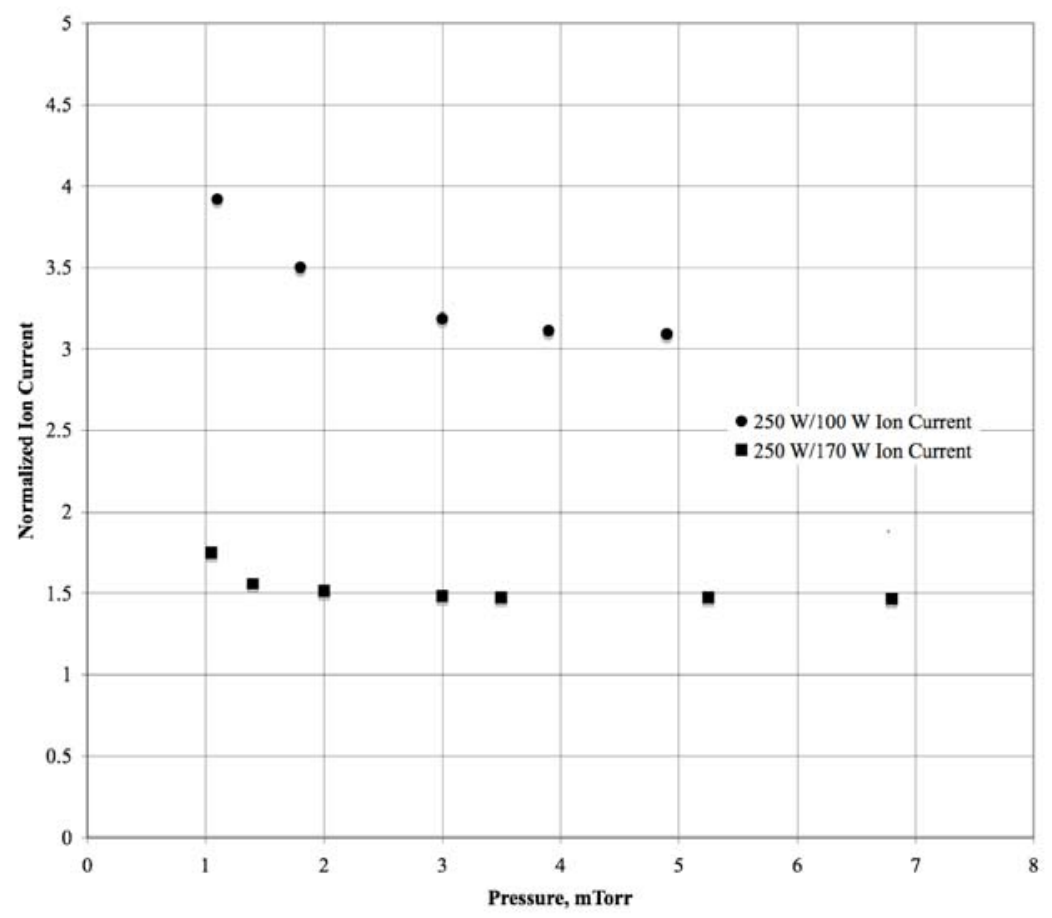

Figure 7. Variation in ion current ratios with pressure.

\section{Ion Production Efficiency}

Using the ion current data, it is possible to calculate the ion production efficiency in discharge Watts per Ampere of ion current collected by dividing the absorbed power by the collected ion current at the location of the disk electrode. Figure 8 depicts the variations in this parameter as a function of pressure. The efficiency of the discharge decreases with pressure. This is a direct result of reduced collisions and reduced coupling as discussed earlier. Each curve has a distinct knee, which tends to shift somewhat to lower pressures with increasing power. The discharge is most efficient at lower pressures at the highest power investigated, $250 \mathrm{~W}$. At 6 mTorr, the efficiency was approximately $250 \mathrm{~W} / \mathrm{A}$ at the $250 \mathrm{~W}$ condition. At $1.3 \mathrm{mTorr}$, the efficiency at $250 \mathrm{~W}$ was observed to drop to 290 W/A. These measurements were all taken with argon. Published data on much larger inductive sources investigated for plasma processing applications indicate comparable ion production efficiency at $1 \mathrm{mTorr}$. Hopwood reports $250 \mathrm{~W} / \mathrm{A}$ at $1 \mathrm{mtorr}$ and similar power levels. Its remarkable that the inductive ion source developed here has comparable performance to the much larger $27 \mathrm{~cm}$ diameter source developed by Coultras and Keller which also features magnetic confinement. ${ }^{23,15}$ Typically systems with large surface area to voltage ratio perform considerably poorer than larger sources with a lower surface area to volume ratio. ${ }^{25}$ The comparable performance of this smaller source to the larger source is a consequence of the magnetic circuit geometry. Additionally, as mentioned earlier, it is expected that operation on xenon should result in significant increases in efficiency owing to the decreased ionization potential of this atom.

Ion production efficiency at the $170 \mathrm{~W}$ and $250 \mathrm{~W}$ cases indicated comparable performance over a wide range at the higher pressures. This data seem to indicate saturation in the discharge efficiency at higher pressures. Significant deviations are apparent however at the lower pressures. Here, the $250 \mathrm{~W}$ operating condition is more efficient. Apparently at the lower pressures, $\mathrm{rf}$ coupling to the plasma improves with increasing power. The behavior of the $100 \mathrm{~W}$ case is consistent with this assertion.

It is also possible to estimate the discharge efficiency if the extraction plane was closer to the antenna. For example, the ion current density can be estimated in the plane of the Langmuir probe. The probe is located approximately $20 \mathrm{~mm}$ above the ion collection electrode. It has been shown that the ion density profile for magnetically confined inductive discharges is essentially flat. ${ }^{9}$ In this regard, the centerline current density can be used to estimate a lower limit on ionization efficiency in this plane as well provided the profile is flat. It is expected that the ion flux closer to the heating zone should result in higher currents and discharge efficiency. 
These calculations were performed and plotted in Figure 9 for the best performing condition, $250 \mathrm{~W}$. These data suggest that optimum placement of the collection plate or ion grid in this case as well as magnetic circuit optimization is key to achieving high performance. As can be seen, the discharge efficiency all the way down to $1 \mathrm{mTorr}$ is less than $200 \mathrm{~W} / \mathrm{A}$. The ion efficiency numbers calculated here are not inconsistent with optimized inductive sources developed for plasma processing applications which have been reported to range from 100 to $300 \mathrm{~W} / \mathrm{A} .{ }^{24}$ These data suggest a pathway to even more efficient ion sources for low power to medium power ion thruster applications. If these efficiencies can be realized in a thruster configuration, then they would not only exceed performance of the barrel-type ion thruster ${ }^{25}$ but would also exceed performance of conventional hollow cathode sources such as previously investigated $8 \mathrm{~cm}$ ion thrusters whose discharge performance ranged between 250 and $350 \mathrm{~W} / \mathrm{A}^{26}$

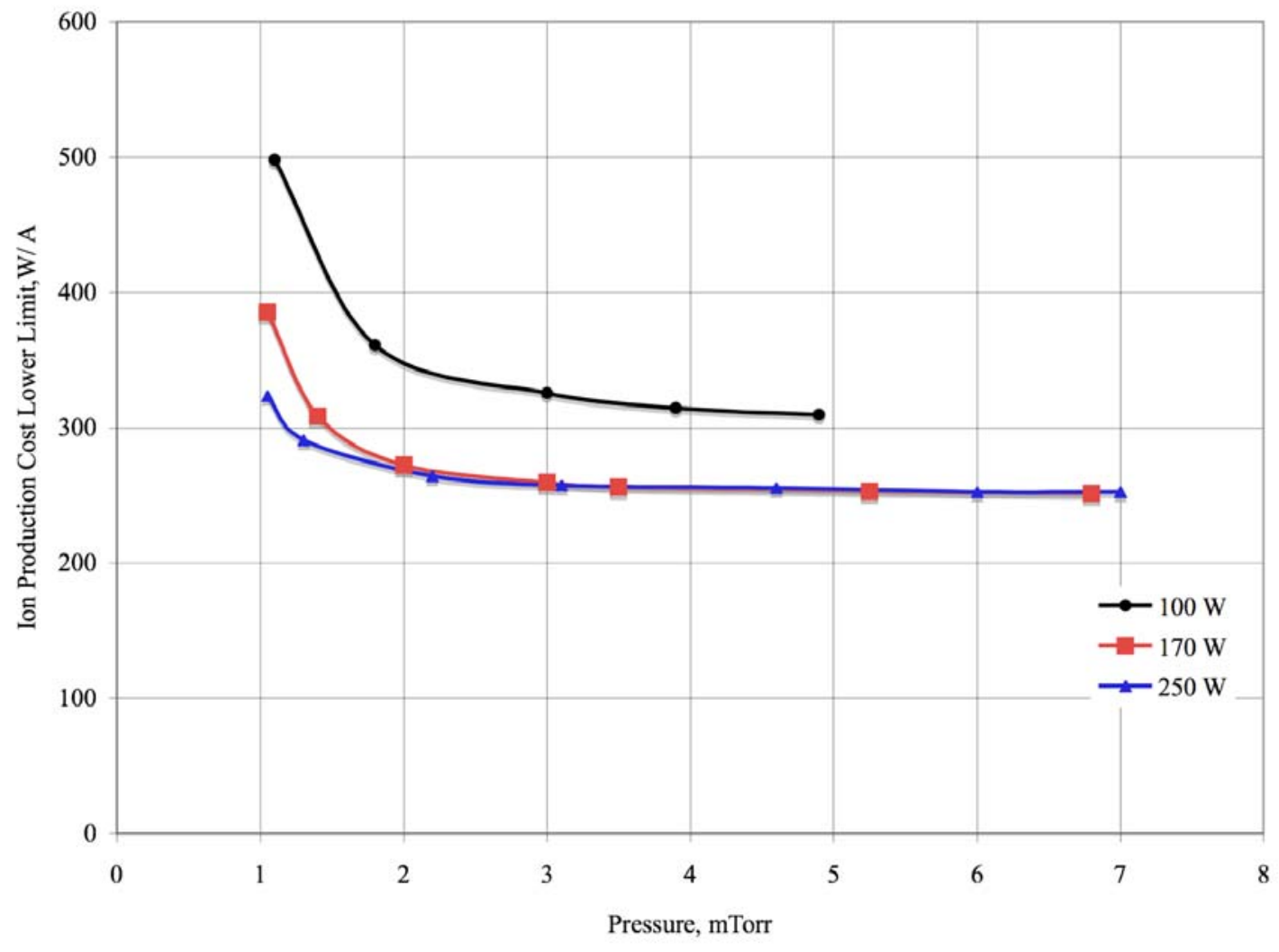

Figure 8. Estimate of ion production efficiency at the exit plane of the source. 


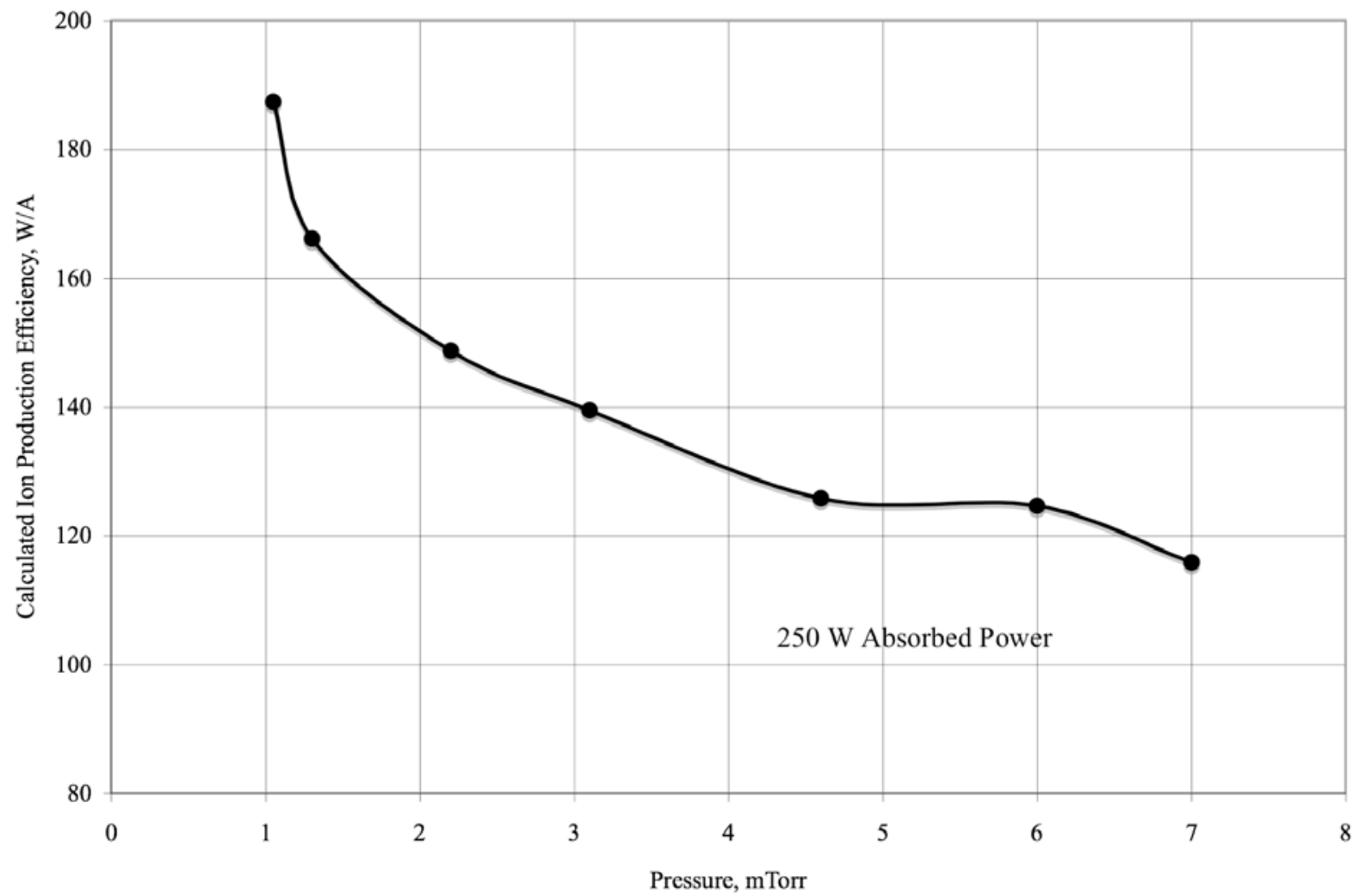

Figure 9. Calculated ion production efficiency in the plane of the Langmuir probe.

\section{Conclusions}

A magnetically enhanced inductive discharge was investigated for potential electric propulsion applications. The source is envisioned for service as the primary ionization stage for small diameter ion thrusters $(<15 \mathrm{~cm})$ or as a stand alone plasma source for an ambipolar accelerator. The inductive source features a compact planar antenna that allows for very low aspect ratio thrusters. Ion production efficiencies were measured as low as 250 W/A at low pressures on argon. Pressures around $1 \mathrm{mTorr}$ are consistent with thruster flows and beam currents approaching 1A at propellant utilizations $>90 \%$. It was found that at low pressures $\sim 1 \mathrm{~m}$ Torr ion production efficiency improved with input rf power. This is speculated to be related to the rf coupling efficiency. At low powers and pressures, capacitive coupling reduces ionization efficiency. A Faraday shield can minimize such coupling. It is not clear however that elimination of the capacitive coupling will bring about improved performance at lower powers and pressures as the capacitive fields though inefficient from a plasma productions standpoint may be necessary for discharge maintenance under these conditions. Magnetic circuit optimization will be necessary to better utilize these fields as well as improve inductive coupling at the lower powers and pressures. Projected ionization efficiency $\sim 2$ $\mathrm{cm}$ downstream of the antenna was estimated using an on centerline Langmuir probe. It was found that ion production efficiencies less than $200 \mathrm{eV} /$ ion may be attainable over the pressure range investigated which extended down to 1 mTorr on argon. Performance of the source on xenon is expected to yield a significant performance improvement. Based on the analysis of the data presented here, the preliminary projected discharge performance appears to be comparable to if not somewhat better than ion thrusters of similar size such as the RIT 10 or the NASA 8-cm. This is remarkable for an unoptimized source and therefore shows promise for future development. Future work will entail a spatial plasma mapping, magnetic circuit optimization as well as the fabrication of a standalone source. Once the operating envelope has been fully assessed of the standalone unit, concrete comparisons between it and existing sources can be made. Ion energy profiles will also be characterized at the exit plane of this device. While much of the initial focus of this work as been on the operation of the device as an ion source, if the 
exiting ion energies are controllable, then the device may have application as a standalone ambipolar thruster as well. Near term work will address this possibility.

\section{Acknowledgments}

The authors would like to acknowledge Aimee Covert, Brett Rogers, and Adam Dow for assistance in carrying out some of the measurements that went into this work.

\section{References}

\footnotetext{
${ }^{1}$ Groh, K.H. and Loeb, H.W., Rev. Sci. Instrum. 65 (5), May 1994, 1741.

${ }^{2}$ Notarantonio, A., Killinger, R., and Amorosi, L., "Ion Propulsion System Saves ARTEMIS Satellite," 54the International Astronautical Congress of the International Astronautical Federation, Bremen, Sept. 29-3, 2003.

${ }^{3}$ Sengupta, A., Brophy, J.R., and Goodfellow, K.D., "The Extended Life Test of the Deep Space 1 Flight Spare Ion Engine after 30,352 Hours of Operation," AIAA-2003-4558.

${ }^{4}$ Hansen, J.W., "U.S. TWTs form 1 to $100 \mathrm{GHz}$," Microwave Journal, 1989, Vol. 32, pp. 179-183.

${ }^{5}$ Kuninaka, H., Nishiyama, K., Funaski, I., Yamada, T., Shimizu, Y., and Kawaguchi, J., "Powered Flight of Hayabusa in Deep Space," Proceedings of the $42^{\text {nd }}$ AIAA Joint Propulsion Conference, July, Sacramento, AIAA Paper 2006-43118, 2006.

${ }^{6}$ Rayman, M.D., Vaghese, P., Lehman, D.H., and Livesay, L.L., “ Results from the Deep Space 1 Technology Validation Mission," Acta Astronautica, Vol. 47, 475, 2000.

7 Koppel, C.R., Marchandise, F., Prioul, M., Estublier, D., and Darnon, F., "The SMART-1 Electric Propulsion Subsystem around the Moon: In Flight Experience," AIAA 2005-3438, Proceedings of the $41^{\text {st }}$ Joint Propulsion Conference, July 2005.

${ }^{8}$ Groh, K.H. and Loeb, H.W., Rev. Sci. Instrum., 65 (3) May 1994, 1741.

${ }^{9}$ Liberman, M.A. and Lichtenberg, A.J., Principles of Plasma Discharges and Materials Processing, John Wiley and Sons, 1994.

${ }^{10}$ Hopwood, J., Plasma Sources Sci. Technol., 1 (1992) 109.

${ }^{11}$ Hopwood, J. et al., J. Vac. Sci. and Technol., A11, 147, (1993).

${ }^{12}$ Manheimer, W.M., and Fernsler, R.F., IEEE Trans. Plasma Sci., Vol. 29, No. 1, Feb. $2001,75$.

${ }^{13}$ Sercel, J., “An experimental and theoretical study of the ECR plasma engine,” Ph.D. Thesis, Cal. Tech. Dept. of Aeronautics and Astronautics, 1993.

${ }^{14}$ Limpaecher, R. and MacKenzie, K.R., Rev. Sci. Instrum. 44, 726, 1973.

${ }^{15}$ Coultas, D.K. and Keller, J.H., 1994, US Patent 5,304,279.

${ }^{16}$ Dickson, M., Zhong, G., and Hopwood, J., J. Vac. Sci. Technol. B 16(2), Mar/Apr 1998, 523.

${ }^{17}$ Sovey, J.S., Dever, J. A., and Power, J.L., Proceedings of the 2001 International Electric Propulsion Conference, IEPC Paper Number 01-86.

${ }^{18}$ Cross, M.F., "Results from Numerical Modeling of RF Ion Thrusters," Proceedings of the $36^{\text {th }}$ JPC, Huntsville, AL., July 2000, AIAA Paper Number 2000-3274.

${ }^{19}$ Olthoff, J.K. and Greenburg, K.E., J. Res. Natl. Inst. Stand. Technol. 100, 327, 1995.

${ }^{20}$ Godyak, V.A. and Popov, O. A., Sov. Phys. Tech. Phys. 22, 4611977.

${ }^{21}$ Groh, K.H., Blum, O., Rado, H., Loeb, H.W., "Inert Gas Radio Frequency Thruster RIT 10," Proceedings of the AIAA International Electric Propulsion Conference, Oct. 30-Nov.1, Princeton, 1979.

${ }^{22}$ Miller, P.A., Hebner, G.A., Greenburg, K.E., Pochan, P.D., and Aragon, B.P., J. Res. Natl. Inst. Stand. Technol. $100,427,1995$.

${ }^{23}$ Hopwood, J., Guarnieri, C.R., Whitehair, S.J., and Cuomo, J.J., J. Vac. Sci. Technol. A 11(1), Jan/Feb., 152, 1993.

${ }^{24}$ Keller, J.H., Plasma Sources Sci. Technol. 5, 1996, 166.

${ }^{25}$ Walther, R.J., Geisel, J.E., Pinks, W.F., Rothermel, K.H., Trojan, F.M., and Loeb, H.W., "Scaling Laws for Radio-Frequency Ion Thrusters," AIAA Paper 75-367, 1975.

${ }^{26}$ Patterson, M.J., “Low Power Ion Thruster Development Status,” NASA TM 199-208822, 1999.
} 